\title{
Correlation Method of Measurements of Ion Beam Parameters
}

\author{
A.S.Artiomov \\ Laboratory of High Energies, JINR \\ 141980, Dubna, Russia
}

\section{Abstract}

A correlation method of nonperturbative control on the ion energy spectrum, beam profile and transverse emittance in a bending transport line area, is suggested. The method is based on measurements of the cross-correlation function between a flux of photons or particles from a probing target pseudorandomly modulated in time, and that of fast informationcarrier convoy particles produced in the target and recorded at a drift distance. Characteristics of the apparatus used to realize the proposed diagnostic method by means of time-integrated correlometers based on charge-coupled devices, are considered for a source of $\mathrm{H}^{-}$ions.

\section{INTRODUCTION}

For high-brightness ion accelerators it is important to obtain information on beam parameters not affecting them appreciably during measurements (nonperturbative diagnostics). For this purpose in a bending transport line area fast neutral or charged convoy particles can be used. These particles are produced as a result of ion destruction or the charge-exchange process in a specially shaped target which is practically transparent for a beam (for $H^{-}$beams see [1-5]). The target is formed so that these informationcarrier convoy particles (IN-particles) follow the ion velocity in magnitude (in relative units) and in direction (in rad) with accuracies required for measurements. These accuracies can be estimated by $\leq\left(\mu_{o} I_{0} / M_{i} E_{i}\right)^{0.5}$ where $\mu_{o}$ is the reduced mass of the IN-paticle and the remaining part of the ion in its destruction or the ion and electrons in their recombinanion, $I_{o}$ is the affinity energy, $M_{i}$ and $E_{i}$ are the ion mass and energy, respectively. In sources, for example of negative ions, the probability of INparticle generation $(\eta)$ in residual gas can be quite considerable (for the neutral IN-particles $\eta_{r} \approx 0.2-0.4$ ). In this case, using the well known methods [1-5] for any density of a probing target, it is impossible to separate directly information on beam parameters from a flux of background IN-particles on a detector. The correlation method of nonperturbative measurements of ion beam parameters considered in this paper allows one to overcome these difficulties.

\section{METHOD}

The correlation method of nonperturbative measurements of the ion energy spectrum has been previously proposed $[6,7]$. It is based on the use of test IN-particles produced in a target, pseudorandomly modulated in time, and detected at drift distance $L$. To measure the transverse beam emittance, for example in the $\left(Y, Y^{\prime}\right)$-plane (see Figure), one or a few threadtype targets parallel to the $(X, Z)$-plane can be formed in front of a bending transport line area. If the ion beam current is invariable during measurements, the spatial $X$-dimension of the target must be required for reproducing target time modulation by the flux $\Psi_{p n}^{\circ}$ of test INparticles

$$
\Psi_{p n}^{o}(t)=\operatorname{const}_{(n)} \cdot \Gamma_{n}^{\gamma}(t),
$$

where $I_{n}^{\gamma}$ is the flux of photons or particles in the $n$-target. The targets are fixed in space 
and separated from each other along the $Y$-axis. When one target is used, it moves in parallel along the $Y$-axis. Taking into account (1), the autocorrelation function of the flux of test INparticles on a $m$-detector is equal to

$$
\begin{aligned}
R_{n m}^{p p}(\tau) & =\int_{-\infty}^{+\infty} \Psi_{p n}^{o}(t) \cdot \Psi_{p n}^{o}(t-\tau) d t= \\
& =\sum_{k=-\infty}^{+\infty} \delta(\tau-k T) .
\end{aligned}
$$

The palsed characteristic $h_{n m}(t)$ of the drift distance from the $n$-target to the $m$-band-type detector $\left(Y^{\prime}=f i x\right)$ is related to the velocity $(V)$ distribution of IN-particles $(t=L / V)$ in the $n-m$ direction and, hence, to the energy spectrum of ions. The fluxes of IN-particles in the $n$-target area $\left(\Psi_{n}^{o}\right)$ and on the $m$-detector $\left(f_{n m}^{o}\right)$ are related by the convolution

$$
\begin{gathered}
f_{n m}^{o}(t)=C_{m} \cdot \int_{0}^{+\infty} h_{n m}(\tau) \cdot \Psi_{n}^{o}(t-\tau) d \tau, \\
\sum_{m} C_{m}=1,
\end{gathered}
$$

where $\Psi_{n}^{\circ}=\Psi_{b n}^{\circ}+\Psi_{p n}^{o}, \Psi_{b n}^{\circ}$ is the flux of background IN-particles produced in the residual gas. Taking into account the independence of $\Psi_{b n}^{o}$ and $\Psi_{p n}^{o}$ and measuring the crosscorrelation function between the fluxes of target particles or photons and IN-particles on the detector

$$
\begin{gathered}
R_{n m}^{\gamma o}(\tau)=\int_{-\infty}^{+\infty} I_{n m}^{\gamma}(t) \cdot f_{n m}^{o}(t-\tau) d t= \\
=B_{n m} \cdot \int_{0}^{+\infty} h_{n m}(t) \cdot R_{n m}^{p p}(\tau+t) d t= \\
=B_{n m n} \cdot \sum_{k=0}^{+\infty} h_{n m}(\tau-k T),
\end{gathered}
$$

we obtain the pulsed characteristic of the drift distance in the $\mathrm{n}-\mathrm{m}$ direction $\left(I_{n m}^{\gamma} \propto I_{n}^{\gamma}\right)$. Using normalization $\int h_{n m}(\tau) d \tau=1$, we get from the $B_{n m}$-matrix information on the ion distribution in the $\left(Y, Y^{\prime}\right)$-plane and thus on the beam $Y$ profile and transverse emittance.

In reality, we must form such targets when convolution (4) of $h_{n m}$ and $R_{n m}^{p p}$ does not change the supposed $h_{n m}(t)$-function. In accordance with [8], this condition means that a periodically replicating element of the autocorrelation function of the $I_{n}^{\gamma}$-flux must have a sufficiently narrow shape in time with width $\Delta \ll \tau_{m a x}$, where $h_{n m}(\tau)=0$ for $|\tau| \geq \tau_{\max }$, and its period $T$ must meet the condition $T>2 \cdot \tau_{\max }$. Correlation methods measure a useful signal with a background which is several orders of magnitude more than the signal. Thus, measuring $R_{n m}^{\gamma o}(\tau)$ by " $n \times m$ " correlometers, the energy spectrum and distribution of ions in the $\left(Y, Y^{\prime}\right)$ plane can be controlled without perturbation of beam parameters.

\section{APPARATUS}

Nonperturbative measurements of ion beam parameters, for example in a source of $\mathrm{H}^{-}$ions, can be realized according to the scheme shown in Figure. It is analogous to the previously proposed one [7], but it contains " $n$ " identical photon targets $\left(I_{n}^{\gamma}\right)$ and photon detectors $\left(D_{n}^{\gamma}\right), " n \times m$ " correlometers $\left(C_{n m}\right)$ and bandtype detectors $\left(D_{n m}^{\circ}\right)$ of fast $H^{\circ}$ atoms. When probing targets are formed by diaphragming radiation with an optimum polarization and a wavelength of $\lambda=10600 \AA$ from the Nd:YAG laser with synchronized modes (see Fig.a,b), the test $H^{\circ}$ atoms follow the $H^{-}$velocity in magnitude (in relative units) and in direction (in $\mathrm{rad}$ ) with accuracies of $\sim 4 \cdot 10^{-3} \cdot\left(E_{i}[\mathrm{keV}]\right)^{-0.5}$. The series duration of pseudorandom radiation pulses is $T_{s} \approx 100 \mathrm{~ns}$ and the width of the autocorrelation function is $\Delta \approx 50 \mathrm{ps}$. [9]. Thus, such photon targets due to $H^{-}$photodetachment can efficiently generate test IN-particles $\left(H^{\circ}\right)$ and allow one to measure pulsed characteristics of the drift distance $h_{n m}(\tau)$ which are fairly short in time. At present, potentialities of the above diagnostics are mainly limited by the fast action of correlometers. The cross-correlation function $R_{n m}^{\gamma o}(\tau)$ between the 


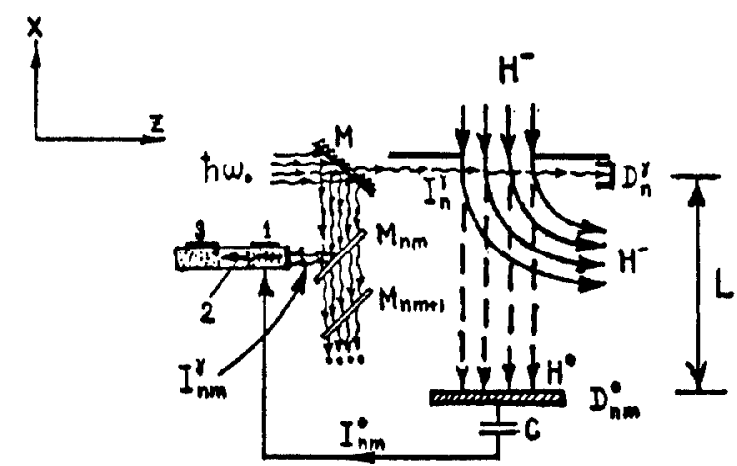

a)

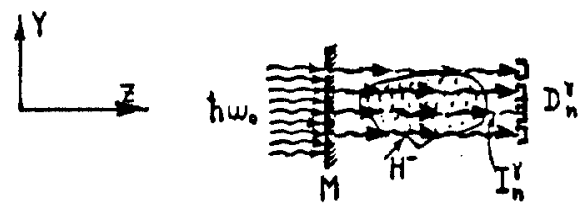

b)

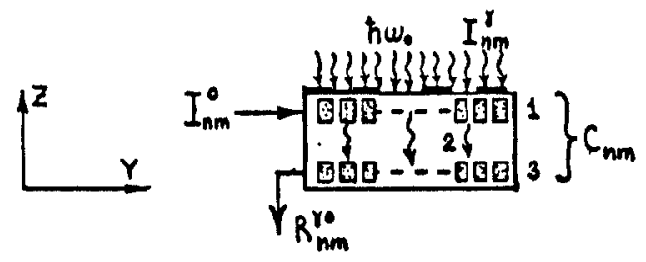

Figure : Schematic of measurement apparatus.

photon flux $I_{n m}^{\gamma}$ from a partly reflecting $M_{n m}$ mirror and current $I_{n m}^{o} \propto f_{n m}^{o}$ from the $D_{n m}^{\circ}$ detector can be measured by means of a timeintegrated $C_{n m}$-correlometer based on chargecoupled (CC) linear structures $[7,10]$. As a result of the wavequide propagation of photons through GaAs CC-linear structure 1 (see Fig.a,c), the $I_{n m}^{o}$-current modulates the flux $I_{n m}^{\gamma}$ by the photoelectric absorption effect within a $\approx 100 \%$ dynamical range of modulation. An instantaneous spatial distribution of charges over the pixels of this structure corresponds to the discrete-in-time representation of the shape of a $I_{n m}^{o}$-current signal. After the modulation, photon flux 2 is detected by silicon CC-linear structure 3. The spatial distribution of charges accumulated there during the measurement time $T_{m}$ corresponds to the discrete-in-time presentation of the $R_{n m}^{\gamma o}$-function. Fairly large $I_{n m}^{\gamma}$-fluxes of photons provide the needed charge within the pixels of the detected CC-linear structure du- ring a short time within a pulse of the ion beam. The $R_{n m}^{\gamma o}$-functions can be read out during intervals between target switchings or between ion beam pulses and taking into account the guiding frequency of modulating structures 1 .

As estimates for the source of $\mathrm{H}^{-}$ions with an energy of $E_{i} \approx 20 \mathrm{keV}, L \approx 100 \mathrm{~cm}$ and the average power density of laser radiation within the duration of a series of pulses $I_{n}^{\gamma} \approx 4 \cdot 10^{5} \mathrm{~W} / \mathrm{cm}^{2}$ $\left(\Psi_{b n} / \Psi_{p n} \approx 10^{2}\right)$, the proposed apparatus allows one to realize nonperturbative measurements of beam parameters during $T_{m} \geq T_{\mathrm{a}} \approx$ 100 ns with accuracies, e.g., of $\approx 0.4 \%$ in energy and of $\approx 2 \cdot 10^{-4} \mathrm{rad}$ in $Y^{\prime}$.

\section{References}

[1] Stephen L.Kramer, D.Read Moffett, IEEE Trans. Nucl. Sci., NS-28, 2174 (1981).

[2] W.B.Cottingame et al., IEEE Trans. Nucl. Sci., NS-32, 1871 (1985).

[3] K.Saadatmand, K.Johnson, J.Schneider, IEEE Part. Accel. Conf., San Francisco, California, May 6-9, 1991, Vol.3, p.1183.

[4] R.C.Connolly, D.P.Sandoval, ibid, p.1237.

[5] R.C.Connolly et al.,Nucl. Instr. Meth. A, Vol.312, p.415 (1992).

[6] A.S.Artiomov, Avtor. Svid. SSSR No.298206, G01T1/36, 1988.

[7] A.S.Artiomov, IEEE Part. Accel. Conf., San Francisco, California, May 6-9, 1991, Vol.3, p.1576.

[8] Methodes et techniques de traitement du signal et applications aux mesures physiques. Tome 1, par J.Max, Paris, 1981.

[9] Ultrashort Light Pulses (picosecond techniques and applications), Edited by S.L.Shapiro, New York, 1977.

[10] R.H.Kingston, Proc. IEEE, Vol.72, p.954 (1984). 